\title{
Tw'o-Dimensional Finite Element Analysis of a Polymer Gel Drug Delivery System
}

\author{
Daniel J. Segalman and Walter R. Witkowski
}

\author{
To be presented at the \\ International Conference on Intelligent Materials \\ Williamsburg VA \\ June 5-8, 1994
}

\section{DISCLAIMER}

This report was prepared as an account of work sponsored by an agency of the United States Government. Neither the United States Government nor any agency thereof, nor any of their employees, makes any warranty, express or implied, or assumes any legal liability or responsibility for the accuracy, completeness, or use iulness of any information, apparatus, product, or process disclosed, or represents that its use would not infringe privately owned rights. Reference herein to any specific commercial product, process, or service by trade name, trademark, manufacturer, or otherwise does not necessarily constitute or imply its endorsement, recommendation, or favoring by the United States Government or any agency thereof. The views and opinions of authors expressed herein do not necessarily state or reflect those of the United States Government or any agency thereof. 


\section{ABSTRACT}

Hydrogels are being investigated as drug delivery mechanisms. Gels can be impregnated with a drug and then stimulated through various means to release it. Having the capability to numerically predict the dynamic behavior of the release process would benefit the design and control of the such a process. In this paper, a finite element analysis is used to simulate the dynamic behavior of an eroding polyelectrolyte gel. The gel is impregnated in a collapsed state. It is then subjected to a higher $\mathrm{pH}$ environment causing it to swell. When it has swollen to a specified extent, the gel erodes, thereby releasing the drug agent. Such gels are currently being investigated in drug delivery schemes to the colon.

\section{INTKODUCTION}

Time released delivery of drugs is a very important technique in the administration of medication. Such methods allow for a therapeutically desirable concentration to be sustained as a function of time. Researchers are currently investigating more efficient and safer techniques than conventional oral and intravenous delivery methods.

Polymeric gels have been investigated for medical applications for the last twenty years. Some hydrogels have shown great potential as the drug release reservoir in drug delivery systems (DDS). At least three different DDS schemes have been investigated. In the first, a thermosensitive gel is used to control the rate of drug release as the environmental temperature is changed. The second uses a chemo-sensitive gel whose diffusion properties are sensitive to a chemical concentration in its environment. The third uses an erodible gel to disseminate the internally contained drug. Experimentation has shown very successful results for each of these schemes.

Having the capability to numerically predict the dynamic behavior would be very valuable in the design phase of these systems. In addition to drug release performance, real-time control strategies can be evaluated. This paper describes how a method of weighted residuals has been used to solve the two-dimensional system of governing equations by finite element analysis.

Formulation of a mathematical model that accurately describes the dynamic behavior of the gel requires proper accounting for the swell or contraction of the polymer network, the fluid

Both members of technical staff in Structural Dynamics and Vibration Control Dept., Sandia National Laboratories. This work was performed at Sandia National Laboratories supported by the U. S. Department of Energy under contract DE-AC04-94AL85000. 
transfer into and out of the gel, and the coupled effects between the two phenomena. Also, large deformation kinematics must be used. The interaction between solvent and gel requires the use of two internal state variables to completely describe the system. Complete dynamic model descriptions have been offered by Grimshaw et al. (1990) and Segalman et al. (1992a).

Theoretical predictions based on a finite element analysis solution scheme were first investigated by Segalman et al (1992b, 1993). They solved the one and two-dimensional, dynamic analysis problem of a swelling and contracting gel sphere and disk, respectively.

In this presentation, theoretical results of the eroding drug delivery system are shown. Computational results of drug release rates and other important states are presented. These calculations provide some insight into the mechanisms of these systems and their relative advantages.

\section{THEORETICAL MODEL OF GEL DYNAMICS}

A slightly modified form of the theoretical model presented in Segalman et al.(1992) is used in this analysis. Modifications include representing the solvent concentration in terms of a mass fraction instead of a density value. In this formulation, the mass transport relationships and the elasticity cquations are cast in a Lagrangian, or convected, framework.

To properly describe the polyelectrolyte gel/solvent system, three components (polymer, solvent, and hydrogen ions) must be accounted for. It is often convenient to express concentration states in terms of mass fraction. In the solution of these relationships, since the mass fraction of hydrogen ions is always small, the mass fraction of the polymer component is simply calculated as one minus the mass fraction of solvent. Using this assumption, the solvent concentration, $c_{s}$, is expressed as a function of $\xi_{s}$, the mass fraction by:

$$
c_{s}=g\left(\xi_{s}\right)=\frac{\rho_{p} \xi_{s}}{1-\xi_{s}\left(1-\rho_{p} / \rho_{s}\right)}
$$

where $\rho_{p}$ is the mass density of pure polymer and $\rho_{s}$ is the mass density of pure solvent. (The mass fraction of polymer and hydrogen ions are represented by $\xi_{p}$ and $\xi_{H}$, respectively.)

Recall since there are three system components, two independent diffusion equations must be formulated with each involving the gradients of at most two of the components. The isothermal diffusion equations describing the evolution of solvent and hydrogen ion concentration in a Eulerian, or fixed, framework are

$$
\begin{gathered}
\frac{\partial c_{s}}{\partial t}=\nabla \cdot\left(\widehat{D}_{s p}\left(c_{s}, c_{H}\right) \nabla c_{s}+\widehat{D}_{H s}\left(c_{s}, c_{H}\right) \nabla c_{H}\right)-\nabla \bullet\left(c_{s} v\right) \\
\frac{\partial c_{H}}{\partial t}=\nabla \cdot\left(\widehat{D}_{H}\left(c_{s}, c_{H}\right) \nabla c_{H}\right)-\nabla \bullet\left(c_{H} v\right)+\dot{H}
\end{gathered}
$$


where $v(x, t)$ is the gel velocity at location $x$ and time $t$, and $c_{s}\left(r_{i} t\right)$ is the mass of solvent per unit volume of gel at that time and position and $c_{H}(x, t)$ is the mass of hydrogen ions per unit volume of gel at that time and position. In terms of the solvent mass fraction, $\xi_{s}$, and the hydrogen mass fraction, $\xi_{H}$, the equations become

$$
\begin{gathered}
\frac{\partial \xi_{s}}{\partial t}=\nabla \bullet\left(D_{s p}\left(\xi_{s}, \xi_{H}\right) \nabla \xi_{s}+D_{H s}\left(\xi_{s}, \xi_{H}\right) \nabla \xi_{H}\right)-\nabla \bullet\left(\rho_{g} \xi_{s} v\right) \\
\rho_{g \bar{\partial}_{t}}=\nabla \cdot\left[D_{H}\left(\xi_{s}, \xi_{H}\right) \nabla\left(\rho_{g} \xi_{H}\right)\right]-\nabla\left(\rho_{g} \xi_{H}\right) \bullet v+\rho_{g} \dot{\xi}_{H_{s}}
\end{gathered}
$$

where $\rho_{g}$ is the current density of the gel. Note the inclusion of source terms for $\mathrm{H}^{+}$ions. Through the source term a tunable parameter is available that may be varied through electrical or chemical means.

The diffusion relationships are taken from Flory-Huggins Theory (Flory, 1969). The hydrogen diffusion coefficient is represented as

$$
D_{H}=D_{H}^{T}\left(\frac{1-\xi_{p}}{1+\xi_{p}}\right)^{2} \approx 10^{-5} \xi_{s}^{2}
$$

The diffusion coefficient for the solvent in the polymer is given as

$$
D_{s p}=\frac{L_{s p}}{\xi_{p}^{0.5}}\left[(1-2 \chi)-\frac{1}{3 N}\left(\frac{\xi_{0}}{\xi_{p}}\right)^{0.667}+\frac{K_{a}}{K_{a}+53 \xi_{H}}\right]
$$

The hydrogen diffusion coefficient through solvent is formulated as

$$
D_{H s}=\frac{-L_{s p}}{\xi_{p}^{0.5}}\left[\frac{K_{a} \xi_{p}}{\left(K_{a}+53 \xi_{H}\right)^{2}}\right]
$$

The nomenclature for material parameters used above is that of Flory: $\chi$ is the FloryHuggins interaction parameter, $N$ is the number of effective cross-links, $L_{s p}$ is the Onsager coefficient and $\mathrm{K}_{\mathrm{a}}$ is the disassociation coefficient.

Transforming the diffusion relationships from a fixed frame into a convective frame involves defining concentrations at gel particles rather than at positions in space. For reasons of clarity, the kernel of the process of transforming diffusion equations from Eulerian to Lagrangian frames is presented for the case of diffusion of a single component; the process is identical for multi-component systems.

In this development, the transformation is done in terms of concentrations and then converted into mass fractions at the end. If each particle of gel is labelled by its original position 
$\boldsymbol{X}$ at time $t_{0}$, movement of the gel at any time, $t$, is determined by the spacial coordinate $\boldsymbol{X}(\boldsymbol{X}, t)$. The moving frame solvent concentration, $C_{s}(\boldsymbol{X}, t)$, is redefined in a convected viewpoint by

$$
C_{s}(X, t)=c_{s}(x(X, t), t)
$$

Note that the two concentration fields are identical at time $t_{0}$. The time derivative of $C_{s}(X, t)$ is evaluated as

$$
\frac{\partial}{\partial t} C_{s}(X, t)=\frac{\partial}{\partial t} c_{s}(x(X, t), t)+\frac{\partial}{\partial x} c_{s}(x, t) \bullet v
$$

Substituting Equation 9 into the Eulerian diffusion equation, the convected form of the diffusion equation is formulated:

$$
\frac{\partial}{\partial t} C_{s}(X, t)=\nabla \bullet\left(D \nabla c_{s}(x(X, t), t)\right)-c_{s}(x(X, t), t) \nabla \bullet v
$$

Using continuity and the assumption of no volume change of mixing, the swell term $\nabla \bullet v$ can be evaluated from:

$$
\nabla \bullet v=\frac{\partial}{\partial t} C_{s}(X, t) /\left(\rho_{s}-C_{s}\right)
$$

Substitution of the swell relationship into the diffusion equation and setting $t_{0}=t$ yields

$$
\frac{\rho_{s}}{\left(\rho_{s}-C_{s}\right)} \frac{\partial}{\partial t} C_{s}(X, t)=\nabla \bullet\left(D \nabla C_{s}(X, t)\right)
$$

Note that the above equation shows that as $C_{s}$ approaches $\rho_{s}$, ever increasing amounts of influx of solvent are required to achieve given increments in solvent concentration. Similarly, the equation for the evolution, in convected frame, of the $\mathrm{H}^{+}$concentration is

$$
\frac{\partial}{\partial t} C_{H}(x, t)=\nabla \bullet\left(D_{H} \nabla C_{H}(x, t)\right)-C_{H} \frac{\frac{\partial}{\partial t} C_{s}(x, t)}{\rho_{s}-C_{s}}
$$

Equation 13 is transformed into terms of mass-fraction through use of Equation 1:

$$
\frac{\rho_{s}}{\left(\rho_{s}-g\right)} g^{\prime}\left(\xi_{s}\right) \frac{\partial \xi_{s}}{\partial t}=\nabla \bullet\left(D g^{\prime}\left(\xi_{s}\right) \nabla \xi_{s}\right)
$$


where $g^{\prime}\left(\xi_{s}\right)=d g / d \xi_{s}$. (This takes on a slightly more familiar form where gradients are small and the term $g^{\prime}$ is distributed out of the divergence and factored from each side.)

The stress relationships for large deformation elasticity require the use of large deformation strain quantities. The deformation gradient $\boldsymbol{F}(t)$ is defined as

$$
F(t)=\frac{\partial x_{g}}{\partial X_{g}}
$$

where $\mathrm{X}_{\mathrm{g}}$ is the location of the particle in the unstrained state. In this problem, it is useful to factor the deformation gradient into its unimodular part and a part representing isotropic swell:

$$
F(t)=F_{u n i}(t) \bullet(\alpha I)
$$

where $\alpha(t)=\operatorname{det}(F(t))^{1 / 3}, \alpha(t)^{3}$ is the volumetric swell of the gel, and $I$ is the identity tensor. The swell is calculated by determining the rate of solvent absorbed. Assuming no volume change of mixing this relationship is

$$
\frac{D \alpha^{3}}{D t}=\frac{\alpha^{3}}{\left(\rho_{s}-c_{s}\right)} \frac{D c_{s}}{D t}
$$

In terms of mass fraction this relationship becomes

$$
\frac{D \alpha^{3}}{D t}=\frac{\alpha^{3}}{\left(\rho_{s}-g\left(\xi_{s}\right)\right)} \frac{d g}{d \xi_{s}} \frac{D \xi_{s}}{D t}
$$

For a solvent-concentration dependent neo-Hookean type solid, the Cauchy stress, $S(t)$, resulting from a given deformation is

$$
S(t)=G\left(c_{s}\right)\left[I-E(t){ }^{T} E(t)\right]-p I
$$

where

$$
E(t)=F_{u n i}(t)^{-1}
$$

$\mathrm{G}$ is the shear modulus, and $\mathrm{p}$ is a Lagrange multiplier dual to the incompressibility constraint on the swollen polymer. Because of the assumed incompressibility of the gel/solvent system, the above equation presents stress only up to an unknown pressure. (Constitutive modeling of rubber-like materials is discussed with good clarity in Lodge (1964).) The incompressibility condition on the swollen polymer is simply a statement that the volume of the material is not a function of the imposed pressure, though volume will swell through a change in solvent concentration.

The conservation of momentum for the gel is 


$$
\rho_{g} \ddot{x}_{g}=\nabla \cdot S+\rho_{g} f_{b}
$$

where $f_{b}$ contains all local body forces, such as gravitational or electromagnetic loads.

\section{Method of Weighted Residuals}

The numerical problem is solved in a fully Lagrangian sense: all field variables are expressed as functions of time, $t$, and of gel particle coordinates $(\zeta, \eta)$. This approach is natural for problems of large deformation, for which the finite element mesh will undergo significant deflection.

The governing differential equations are transformed into a system of algebraic equations through a standard Galerkin-finite element formalism (See Hughes (1987), for instance.) However, because of the Lagrangian formulation, all interpolation is over a material manifold rather than over space. For instance, the configuration field of the gel is interpolated:

$$
x_{g}(\zeta, \eta, t)=\sum x_{g i}(t) \phi_{i}^{x}(\zeta, \eta)
$$

The other fields are also represented as linear combinations of appropriate basis functions:

$$
\begin{aligned}
\hat{\xi}_{s}(\zeta, \eta, t) & =\sum \xi_{s, i}(t) \phi_{i}^{c}(\zeta, \eta) \\
\hat{\xi}_{H}(\zeta, \eta, t) & =\sum \xi_{H, i}(t) \phi_{i}^{H}(\zeta, \eta) \\
\hat{p}(\zeta, \eta, t) & =\sum p_{i}(t) \phi_{i}^{p}(\zeta, \eta) \\
\hat{u}(\zeta, \eta, t) & =\sum u_{i}(t) \phi_{i}^{x}(\zeta, \eta) \\
\hat{x_{0}}(\zeta, \eta) & =\sum x_{0, i}(t) \phi_{i}^{x}(\zeta, \eta)
\end{aligned}
$$

where $\hat{x}_{0}(\zeta, \eta, t)$ is the initial location of particle $(\zeta, \eta)$ and $\hat{u}(\zeta, \eta, t)$ is the displacement of that particle at time $t$ :

$$
\hat{\boldsymbol{x}}(\zeta, \eta, t)=\hat{\boldsymbol{x}}_{0}(\zeta, \eta)+\hat{\boldsymbol{u}}(\zeta, \eta, t)
$$

Gradients are take using the chain rule in the following manner:

$$
\frac{\partial}{\partial x} \hat{\xi}_{s}(\zeta, \eta, t)=\left(\frac{\partial}{\partial(\zeta, \eta)} \hat{\xi}_{s}(\zeta, \eta, t)\right)\left(\frac{\partial}{\partial(\zeta, \eta)} \hat{x}\right)^{-1}
$$


The basis functions for pressure must be one order lower than the basis functions for displacement such that the LBB condition (Hughes 1987) is satisfied, or else some additional constraints must be imposed on the pressure field, as has been suggested by Hughes et. al. (1986). Both methods have been employed successfully in our code, though using the low-order pressure field results in simpler code. Our realization of that simpler form employed quadrilateral elements with linear shape functions for $\phi_{i}{ }^{x}, \phi_{i}{ }^{u}, \phi_{i}{ }^{c}$, and $\phi_{i}{ }^{H}$, each having a value of 1 on node $i$ and a value of 0 on every other node. The basis functions for pressure are taken to be piecewise constant, $\phi_{i}^{p}$ having a value of 1 on element $i$ and a value of 0 on every other element. Note that there are twice as many displacement unknowns as there are nodes and there are exactly as many pressure unknowns as there are elements.

There are as many solvent concentration unknowns and as many $\mathrm{pH}$ concentration unknowns as there are nodes. The weak form of the solvent diffusion equation:

$$
\begin{array}{r}
\int_{A_{i}}\left\{\phi_{i}^{c}(\zeta, \eta)\left(\frac{\rho_{s}}{\rho_{s}-g\left(\xi_{s}\right)}\right) g^{\prime}\left(\xi_{s}\right) \dot{\xi}_{s}+D_{s p} \nabla \xi_{s} \bullet \nabla \phi_{i}^{c}+D_{H s} \nabla \xi_{H} \bullet \nabla \phi_{i}^{c}\right\} \\
\left(\frac{\partial(x, y)}{\partial(\zeta, \eta)} d \zeta d \eta\right)=0
\end{array}
$$

where $A_{i}$ is the support of $\phi_{i}^{c}$ and the gradients are taken with respect to current spacial configuration via Equation 30.

The $\mathrm{pH}$ diffusion equation becomes

$$
\int_{A_{i}}\left\{\rho_{g} \dot{\xi}_{H} \phi_{i}{ }^{H}-\rho_{g} \dot{\xi}_{H_{s}} \phi_{i}{ }^{H}-D_{H} \nabla\left(\rho_{g} \xi_{H}\right) \cdot \nabla \phi_{i}{ }^{H}\right\} \frac{\partial(x, y)}{\partial(\zeta, \eta)} d \zeta d \eta=0
$$

The above two equations yield one scalar equation for each of $\xi_{s}$ and $\xi_{H}$ at every node. A sequential solution strategy was chosen to solve the diffusion equations, but in the examples presented below the $\mathrm{pH}$ field was specified a priori and the solvent field was calculated. Once the mass transfer equations have been solved, these results are piped into the elasticity relationships to calculate the resulting expansion/contraction.

The elasticity equations become:

$$
\int_{A_{i}}\left\{S(\zeta, \eta) \bullet \nabla \phi_{i}^{x}+\left[\ddot{x}_{g}(\zeta, \eta)-f_{b}(\zeta, \eta)\right] \rho_{g} \phi_{i}^{x}\right\} \frac{\partial(x, y)}{\partial(\zeta, \eta)} d \zeta d \eta=0
$$

Note that the above vector-valued equation is equivalent to two scalar-valued equations in the unknown fields $u_{1}, u_{2}$, and $\mathrm{p}$. When displacement is specified at node $i$, the above equation is replaced by the two scalar specifications: 


$$
u_{1}^{i}=u_{1, \text { spec }}^{i} \text { and } u_{2}^{i}=u_{2, \text { spec }}^{i}
$$

Between Equation 32 and Equation 33 there are exactly as many discretized equations as there are displacement unknowns.

The weak form of the incompressibility equation becomes

$$
\int_{E_{i}}\left\{\alpha^{3}(\zeta, \eta)-\frac{\partial(x, y)}{\partial\left(x_{0}, y_{o}\right)}(\zeta, \eta)\right\} \frac{\partial(x, y)}{\partial(\zeta, \eta)} d \zeta d \eta=0
$$

In this equation, $E_{i}$ is the area of element $i$. There are exactly as many distinct equations of constraint as there are unknown pressures.

\section{Drug Delivery Example}

The numerical analysis solution scheme is demonstrated in an eroding gel drug delivery example (see Figure 1). This example mimics similar gel dynamics behavior as that demonstrated
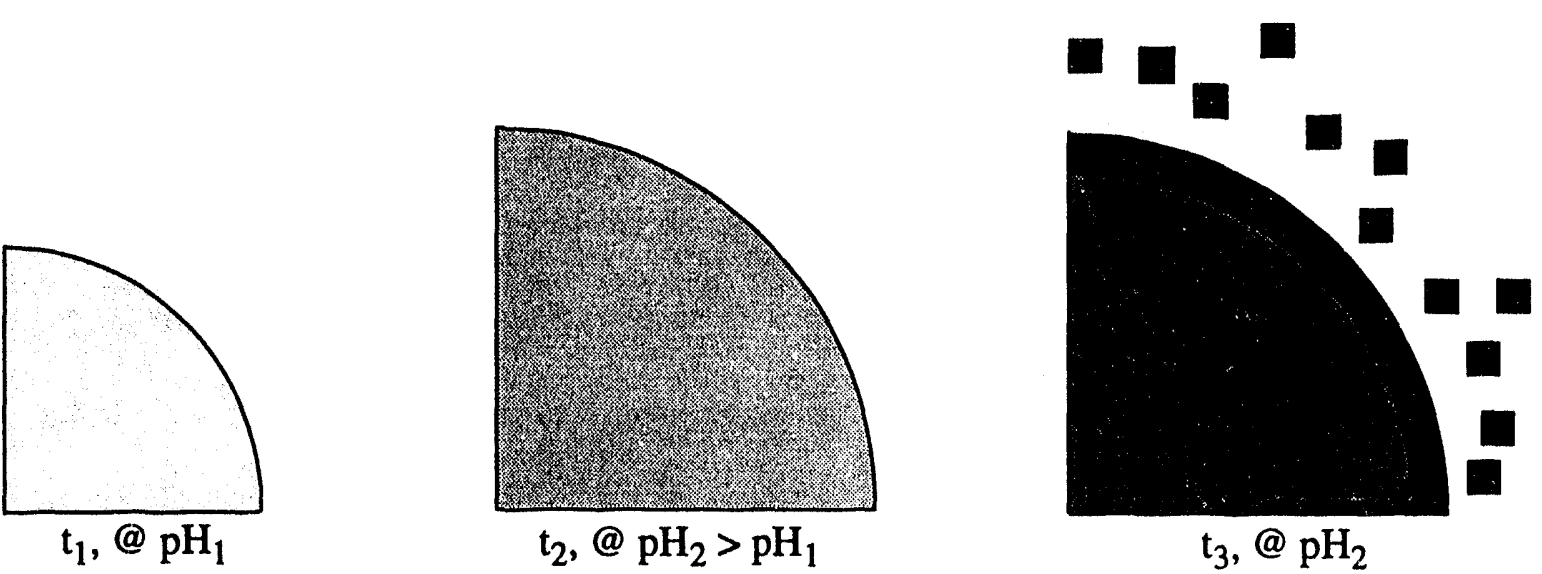

Figure 1: Eroding Drug Delivery Problem - Gel is equilibrated at $t_{1}$ and $\mathrm{pH}_{1}$. At $t_{2}$ gel has swollen in response to $\mathrm{pH}_{2}$ environment. At $\mathrm{t}_{3}$ outer shell of gel has reached the critical concentration for erosion and flakes off.

by the colon drug delivery system described earlier. These doped gels are designed to swell in high $\mathrm{pH}$ environments. At the higher $\mathrm{pH}$ an enzymatic reaction can occur which causes the gel to erode thereby releasing the dopant. In the numerical example an equilibrated gel disk (at $\mathrm{pH}=3$ ) is subjected to changes in its environmental conditions ( $\mathrm{pH}$ increased to 8 ) so that it must swell by absorbing solvent to attain its new equilibrium. These $\mathrm{pH}$ levels are comparable to those the gel would have encountered passing from the stomach into the gastrointestinal tract (actual published $\mathrm{pH}$ levels are 2 for the stomach and 7.4 for the GI-tract (Brondsted and Kopecek,1992). Once the gel has swollen to a certain extent (the criterion is specified by a solvent concentration limit) the gel is eroded. In the numerical problem finite elements are eliminated from the mesh. The geometry of the physical device is a disk. Because of symmetry only a quarter of the gel disk is modeled. The finite element mesh having 147 elements is shown in Figure 2 (along with boundary conditions). The quadrilateral elements are linear in displacement and discontinuous, piecewise constant in pressure. (Calculation with a system of elements that are linear in both 


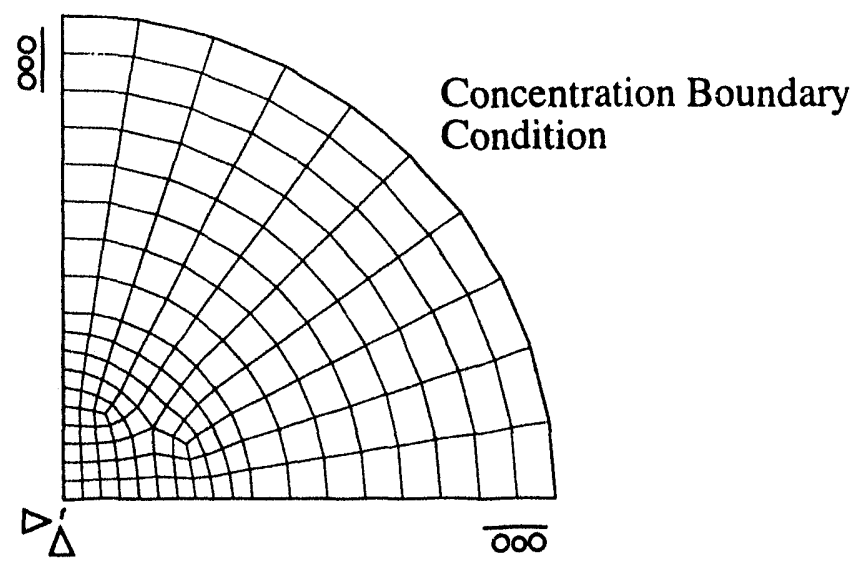

Figure 2: Eroding Drug Delivery Problem - Initial Finite Element Mesh (147 elements).

displacement and pressure and made non-singular by the method of Hughes, Franca, and Balesta (1986) gave results indistinguishable from those presented here.)

In the problem presented, the physical parameters and state variables are chosen to resemble typical values for a polyacrylamide gel system. Initially, the gel is assumed to be equilibrated at a pH of 3 with a corresponding equilibrium solvent mass fraction equal to 0.775 . Then the gel's $\mathrm{pH}$ is changed to 8 (with a corresponding solvent mass fraction equal to 0.989 ). This will cause the gel to absorb solvent and swell. Physical model parameters were derived from experimental observations $\left(\mathrm{K}_{\mathrm{a}}=6 \times 10^{-5}, \chi=0.2, \mathrm{~L}_{\mathrm{sp}}=1 \times 10^{-5} \mathrm{~cm}^{2} / \mathrm{s}\right.$, and $\left.\mathrm{N}=4\right)$. The erosion concentration criterion was set at $\mathbf{0 . 8 5}$. Therefore, when the average concentration of a finite element attained a value of 0.82 it was "eroded" from the mesh.

The change in equilibrium conditions causes the gel to absorb solvent through its outer edge. Therefore, solvent enters first from the outer edge region into the inner region causing concentration gradient rings. Figure 3 shows different solvent concentration profiles of the disk at five different time steps $(2000,3000,4000,5000,5400$ seconds.) As expected, the gel starts to swell until the outer layer solvent concentration reaches the critical limit for erosion where it "flakes" off. Each finite element's solvent concentration value is determined from the average of all of its nodal solvent concentration values. In the numerical analysis program, when the outer layer is removed the boundary condition gets transferred to the new outer layer of gel. When the new outer edge reaches the critical concentration limit it also flakes off. Over time, the inward motion of the boundary accelerates as the diameter of the disk gets smaller and its perimeter/area ratio increases. However, the area of gel that is lost per time step decreases as inward motion of the boundary represents smaller increments of area. Figure $4 \mathrm{a}$ shows the area of the gel disk as a function of time. The discretization of the mesh is reflected in small increases in total area just before the catastrophic loss of a layer of element. This continues until the gel is almost completely eroded. Figure $4 \mathrm{~b}$ shows the area of material lost as a function of time which is related to the dopant release rate. Note that after the initial swell period (before any material is lost) the area lost rate is almost linear. Of course this is dependent on mesh used. We would expect to find the true rate functionality using a very fine mesh density.

As mentioned above, the numerical solution scheme first solves the diffusion calculations and then uses these results in the elasticity calculations to produce a new deformed geometry. 


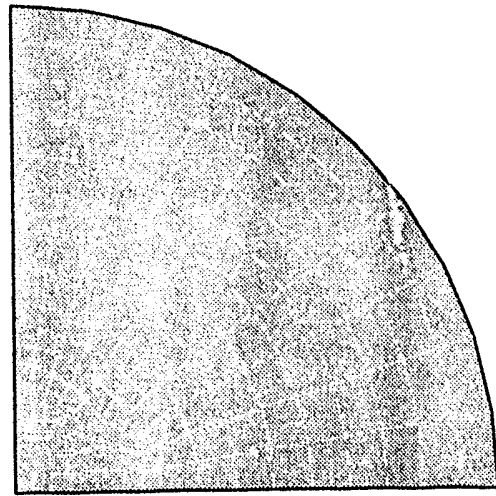

time $=0 \mathrm{sec}$

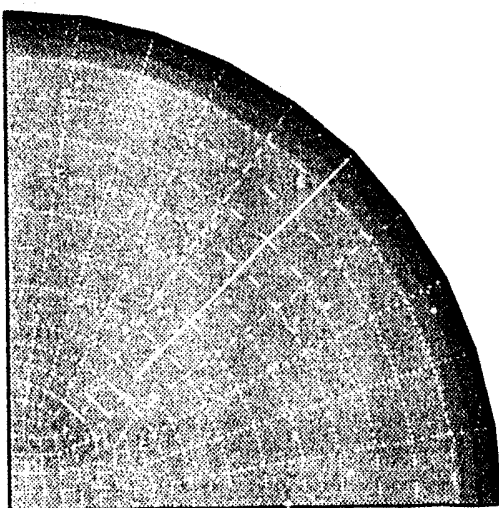

time $=3000 \mathrm{sec}$

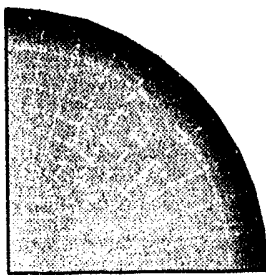

time $=5000 \mathrm{sec}$

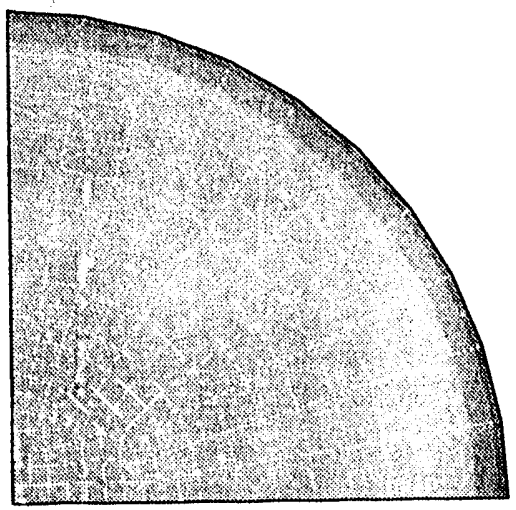

time $=2000 \mathrm{sec}$

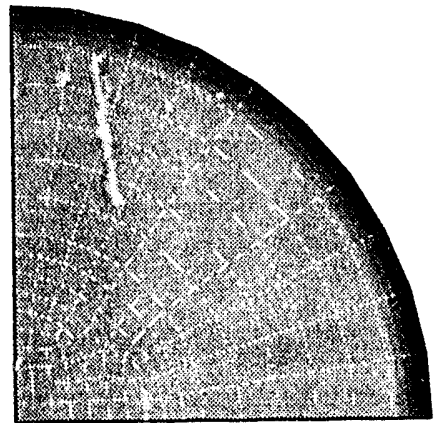

time $=4300 \mathrm{sec}$

c
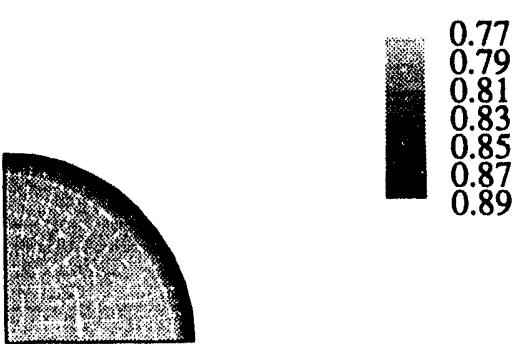

time $=5400 \mathrm{sec}$

Figure 3: Eroding Drug Delivery Problem - Solvent concentration profiles are shown as a function of time. As a finite elements concentration value reaches a critical limit of 0.82 it is removed from the mesh. 


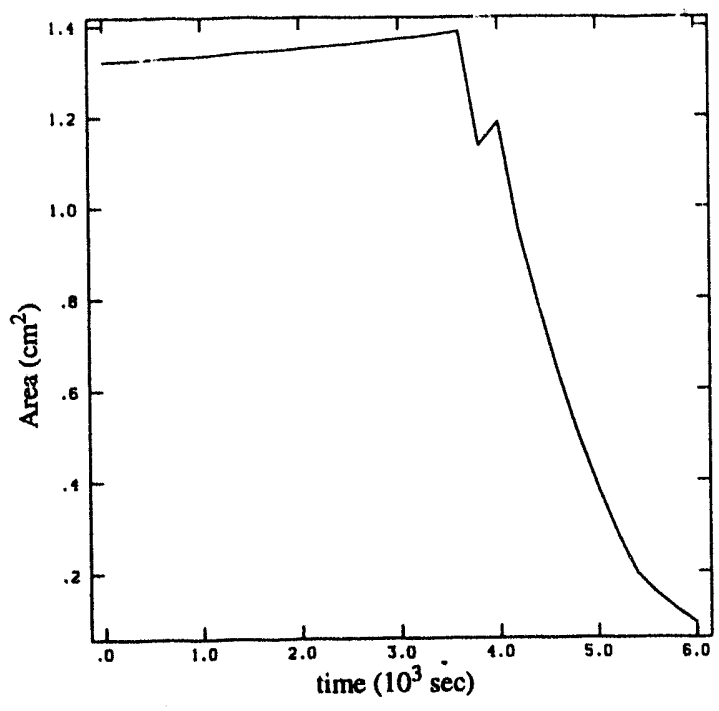

a

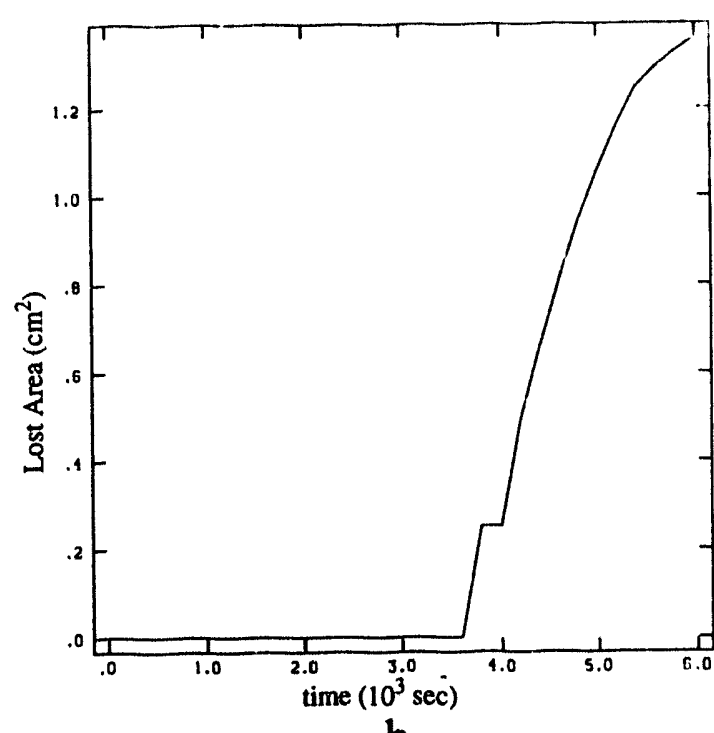

b

Figure 4: Eroding Drug Delivery Problem - 4a shows the area of gel as a function time; $4 b$ shows the area of gel eroded as a function of time.

This can be a numerically unstable procedure if the selected time steps are too large. A time step must be chosen that allows the elasticity to deform the mesh in small amounts. By assuring that the diffusion relationships produce small concentration gradients throughout the gel small changes in swell result. This is done by selecting a time step which is of the same order of magnitude as the rate of diffusion.

\section{CONCLUSIONS}

Polymeric gels are being investigated as drug delivery mechanisms. Gels can be impregnated with a drug and then stimulated through various means to release it. Having the capability to numerically predict the dynamic behavior of the release process would benefit the design and control of such a process. In addition to drug release performance, real-time control strategies can be evaluated.

In this paper a finite element analysis is used to simulate the dynamic behavior of an eroding polyelectrolyte gel. The gel is impregnated in a collapsed state. It is then subjected to a higher $\mathrm{pH}$ environment causing it to swell. When it has swollen to a specified extent the gel erodes, thereby releasing the drug agent. The numerical analysis capability allows the prediction of gel erosion which is related to the drug release rate. Therefore, release rates can be predicted for different dopant loading schemes. This will allow for a larger number of schemes to be investigated. Also, the functionality of how gel properties affect release rates can be studied. Thereby, a new gel can be engineered for a specific release pattern.

\section{REFERENCES}

Brondsted, H. and J. Kopecek, 1992, "pH-Sensitive Hydrogels" in Polyelectrolyte Gels, ed. by Harland and Prud'homme, ACS Symposium Series 480, pp. 285-304.

Flory P.J., 1969, Statistical Mechanics of Polymer Chains, Interscience, New York.

Grimshaw P., J. Nussbaum, A. Grodzinsky and M. Yarmush, 1990, J. Chem. Phys., Vol. 93 No. 6, $4462-4472$.

Hughes T., 1987, The Finite Element Method, Prentice-Hall, Englewood Cliffs, p. 208-209. 
Hughes T., L. Franca, and M. Balestra, 1986, Computer Methods in Applied Mechanics and Engineering 59, pp 8589.

Lodge A., 1964, Elastic Liquids, Academic Piess, New York 62.

Segalman D., W. Witkowski, D. Adolf, and M. Shahinpoor, 1992, Smart Mater. Struct., 1 pp 95-100.

Segalman D., W. Witkowski, D. Adolf, and M. Shahinpoor, 1992, Proc. First Internat. Conf. Intel. Materials, Oiso Japan.

Segalman D., W. Witkowski, R. Rao, D. Adolf, and M. Shahinpoor, 1993, Proc. of Smart Structures and Materials 1993/Smart Materials, SPIE Conf, Vol 1916, pp 14-21. 

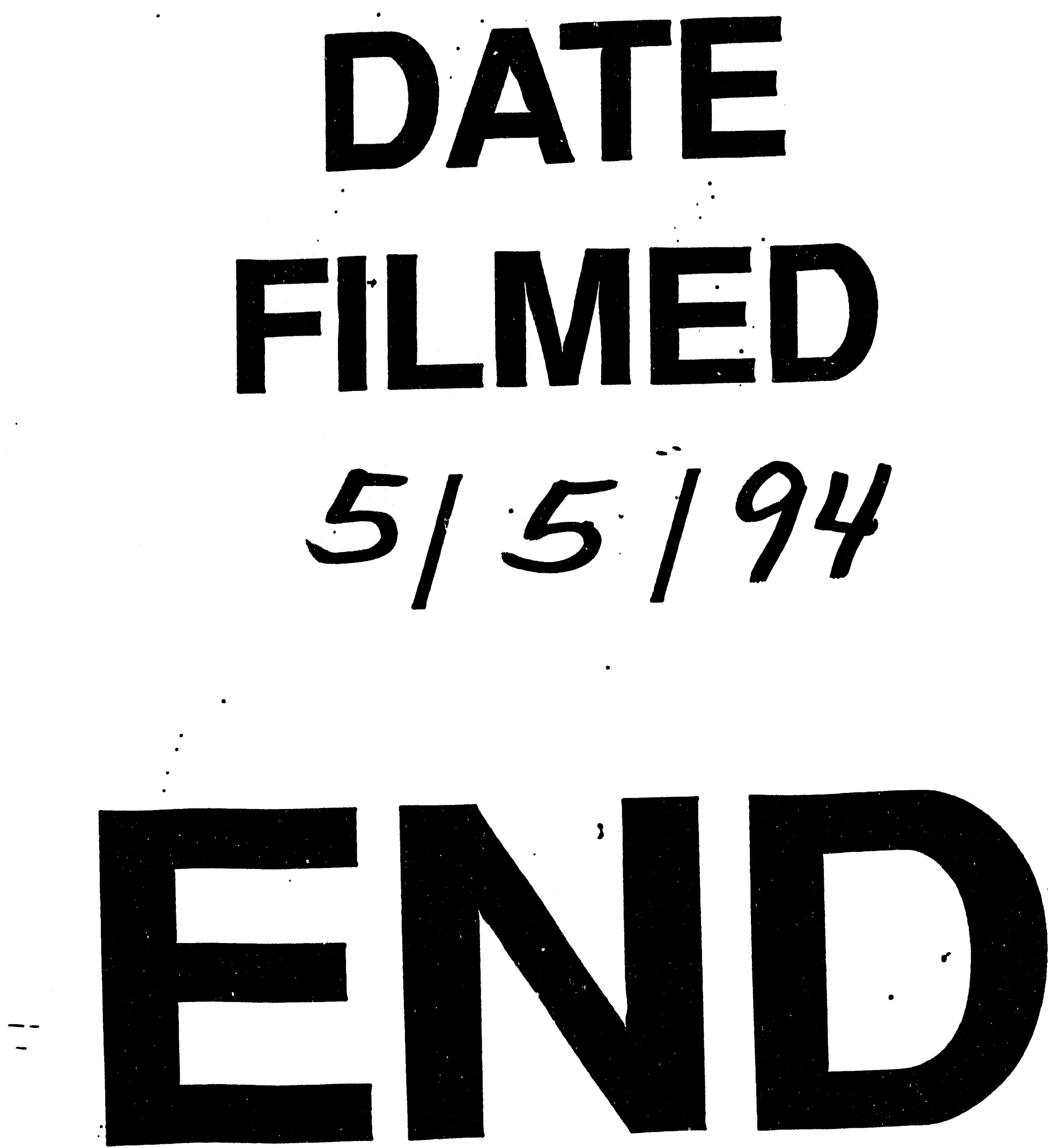
